\title{
Vigas tipo I para la construcción civil fabricadas con madera de plantaciones de rápido crecimiento en Costa Rica
}

\author{
I Beam for civil construction fabricated with fast \\ growing plantation wood in Costa Rica
}

Carolina Tenorio'

Roger Moya²

Mauricio Carranza

Fecha de recepción: 15 de junio del 2013

Fecha de aprobación: 19 de agosto del 2013

Tenorio, C; Moya, R; Carranza, M. Vigas tipo I para la construcción civil fabricadas con madera de plantaciones de rápido crecimiento en Costa Rica. Tecnología en Marcha. Número Especial. Pág 50-59.

I Escuela de Ingeniería Forestal, Instituto Tecnológico de Costa Rica, Cartago, Costa Rica. Tel. (506)2550 2433, Fax (506)259I 3315, Correo electrónico: ctenorio@itcr.ac.cr

2 Escuela de Ingeniería Forestal, Instituto Tecnológico de Costa Rica, Cartago, Costa Rica.

3 Escuela de Ingeniería en Construcción, Instituto Tecnológico de Costa Rica, Cartago, Costa Rica. 


\section{Carolina Tenorio Monge}

Carolina Tenorio Monge, nacida en San José el 16 de marzo del 1983, cursó sus estudios de secundaria en el Colegio Técnico Profesional Industrial de San Sebastián entre los años 1999-200I, donde obtuvo su título de bachiller en educación media y un técnico medio en electrónica, sus estudios universitarios los cursó en el Instituto Tecnológico de Costa Rica, donde se graduó en el 2007 como bachiller en ingeniería forestal y en el 201 I como licenciada en la misma carrera, actualmente cursa la Maestría en Sistemas Modernos de Manufactura de la Escuela de Producción Industrial del Instituto Tecnológico de Costa Rica. Trabajó en el área de industrias forestales como jefe de producción, espe- cíficamente en labores de aserrío y producción de tarimas durante los años 2007-2009. Desde el 2009 se desempeña como asistente de investigación en el área de Tecnología de Productos Forestales de la Escuela de Ingeniería Forestal, donde ha trabajado en siete proyectos inscritos en la Vicerrectoría de Investigación. Sus aéreas de investigación se han concentrado en el estudio de las propiedades físicas, mecánicas y energéticas de la madera de plantación, materiales para la producción de energía o biomasa y el estudio de nuevos materiales a base de maderas de plantación. Actualmente cuenta con más de 15 artículos científicos publicados, dentro de los cuales más de diez han sido en revistas indexadas de alto impacto. 


\section{Palabras clave}

Gmelina arborea; plywood; flexión; cortante; entrepisos; techos.

\section{Resumen}

En años recientes, el mercado de la madera en Costa Rica ha sido abastecido por especies de plantaciones forestales de rápido crecimiento. No obstante, aún se presentan vacíos en el diseño y comercialización de productos de alto desarrollo tecnológico. Con el propósito de introducir las maderas de plantación en el mercado, se estableció como objetivo determinar los esfuerzos de diseño (esfuerzo en flexión, cortante y la rigidez a flexión) de vigas tipo I construidas con madera de Gmelina arborea, utilizando madera sólida en las zapatas y plywood de $12 \mathrm{~mm}$ en el alma. Se desarrollaron tres tipos de perfiles $(24,16$ y $10 \mathrm{~cm}$ de alto) y dos tipos de calidades (A y B). Los resultados mostraron que los esfuerzos de diseño variaron de 60 a 147 $\mathrm{kg} / \mathrm{cm}^{2}$ en el esfuerzo en flexión y de 42 a $92 \mathrm{~kg} /$ $\mathrm{cm}^{2}$ en el esfuerzo en cortante. Las vigas de $10 \mathrm{~cm}$ y de calidad A presentaron los esfuerzos más altos en flexión y cortante, seguidos por las vigas de 24 $\mathrm{cm}$ y $16 \mathrm{~cm}$. Con respecto a la rigidez a flexión, las vigas perfil 24 son las que poseen el valor más alto, con 302787437, I kg-cm²; seguidas de los perfiles 16 y 10. Al demostrar el uso de las vigas en entrepisos, se observó que las vigas de calidad $A$ son las que producen las longitudes óptimas para el mercado. Para techos, la clasificación por calidad de las vigas no produce efectos en la longitud permisible.

\section{Key words}

Gmelina arborea; plywood; bending; shear; flooring systems; roofing systems.

\section{Summary}

Recently, the timber market in Costa Rica has been supplied with fast growing trees from forest plantations. However, there are some problems relationed to design and development of high technology products. In order to introduce the forest plantations woods in the market, the main objective of this study was to determinate the design stress (bending, shear and bending stiffness) from I-beams fabricated with Gmelina arborea wood using lumber for the flanges and $12 \mathrm{~mm}$ plywood for web. Three types of profiles were developed $(24 \mathrm{~cm}, 16 \mathrm{~cm}$ and $10 \mathrm{~cm}$ in high) and two quality types (A and $B$ ). The results showed that the design stress varied from 60 to $147 \mathrm{~kg} / \mathrm{cm}^{2}$ in bending stress and from 42 to $92 \mathrm{~kg} / \mathrm{cm}^{2}$ in shear stress. The I-beams of 10 $\mathrm{cm}$ and A quality showed the highest bending and shear stress, followed by l-beams of the $24 \mathrm{~cm}$ and $16 \mathrm{~cm}$. With regard to bending stiffness, the profile of I-beams of $24 \mathrm{~cm}$ is those with the highest value, with $302787437.1 \mathrm{~kg}-\mathrm{cm}^{2}$, followed by the profiles of $16 \mathrm{~cm}$ and $10 \mathrm{~cm}$. By demonstrating the use of the I-beams in flooring systems, it had been observed that the quality $A$ beams are those that produce optimal lengths for the market. For roofing systems, a quality classification of the beams no effect on the allowable length. 


\section{Introducción}

La creciente demanda de madera a nivel mundial y la incapacidad de los productores de suplir este material de construcción ha permitido el desarrollo de materiales compuestos a base de madera, tales como madera contrachapada, vigas laminadas y vigas I, entre otros (Aydin et al., 2004, Leichti et al., 1990, Maloney 1996). Estos productos permiten darle un uso comercial a trozas de plantaciones, a trozas de bajas calidades y a especies poco utilizadas por su valor comercial o por sus propiedades estructurales (Lam 200 I).

En esa búsqueda de nuevos productos, durante más de 40 años las vigas tipo I se han utilizado como material de construcción, siendo estudiadas para aplicaciones a corto y largo plazo, principalmente en techos y entrepisos (Leicht et al., 1990), en países del norte de Europa y Norteamérica. Este tipo de productos se elaboran a partir de la unión de paneles estructurales de madera (contrachapados laminados) en el alma y madera sólida u otro panel estructural en los extremos (Leicht et al., 1990, Lam 200 I).

Por otro lado, en los países tropicales de América se ha introducido una serie de especies para la reforestación comercial que son poco conocidas a nivel de sus mercados, entre las cuales se destacan Tectona grandis, Gmelina arborea y una gran variedad de Eucaliptus (Moya, 2004; Erskinea et al., 2006). No obstante, el desarrollo de productos con madera de estas especies provenientes de plantaciones se ha visto limitado a productos de bajo desarrollo tecnológico, como madera para tarimas, madera en troza o semielaborada para exportación o bien solamente tableros.

En el caso de la madera de G. arborea, su utilización en materiales compuestos de carácter estructural es muy limitada. Por ejemplo, González et al. (2004) describen el desarrollo del sistema de unión de piezas en una viga laminada para uso estructural. Por su parte, Sasaki et al. (1993) describen las propiedades físicas y mecánicas de tableros tipo LVL de 9 chapas, así como la resistencia de vigas I utilizando madera de G. arborea en sus zapatas. En Costa Rica, Tenorio et al. (20I I) determinaron algunas de las propiedades físicas y mecánicas de tableros de plywood y de LVL manufacturados con chapas de G. arborea provenientes de plantaciones forestales y encoladas con resina de fenol-formaldehído.
De tal forma que el objetivo de este trabajo fue determinar los esfuerzos de diseño de vigas tipo I elaboradas con madera de G. arborea proveniente de plantaciones forestales de rápido crecimiento para ser comercializadas y utilizadas en las construcciones del mercado de Costa Rica. Se determinaron los valores de resistencia en flexión, así como los esfuerzos permisibles y de diseño para vigas I de $G$. arborea de dos calidades y tres perfiles o peraltes diferentes. Finalmente, se utilizan los valores para determinar la longitud máxima permisible para utilizar este tipo de viga en entrepisos y techos de construcciones civiles.

\section{Materiales y métodos}

\section{Materiales}

Las vigas tipo I utilizadas en este estudio fueron construidas por la empresa Maderas Cultivadas de Costa Rica S.A. (www.maderascultivadas.com). Se elaboraron con madera de G. arborea proveniente de plantaciones de rápido crecimiento de las regiones Norte y Sur de Costa Rica. La madera se obtuvo de árboles provenientes del tercer raleo y la cosecha final.

\section{Diseño}

Las vigas I fueron construidas con un ancho de 6,5 $\mathrm{cm}$ y tres alturas diferentes, una de $10 \mathrm{~cm}$ (Perfil I), otra de 16,5 cm (Perfil 2) y finalmente una de 24,2 cm (Perfil 3) (figura I). Las zapatas se diseñaron con madera sólida de diferentes alturas para cada perfil, de 2,8 para los perfiles I y 2 y 3,8 cm para el perfil 3 , con el fin de tener una mejor estabilidad de la viga durante su uso. El alma de las vigas se construyó con madera contrachapada de $12 \mathrm{~mm}$ de espesor.

\section{Proceso de fabricación}

El proceso de fabricación del contrachapado utilizado como alma de las vigas se detalla en Tenorio et al. (20 I I y 20 I2). Se utilizaron dos láminas de I,78 $\mathrm{m}$ en los extremos de la viga y una de $2,44 \mathrm{~m}$ en el centro (Figura Ic), unidas mediante uniones tipo dedo (Figura Ib), de forma tal que no existieran uniones en el centro de la viga. La madera utilizada en las zapatas provenía de tablas de 32 y $42 \mathrm{~mm}$ de espesor, las cuales se secaron a un 12\% de contenido de humedad. Esta madera fue cortada en anchos de $7 \mathrm{~cm}$ para posteriormente eliminar los nudos 
(a)

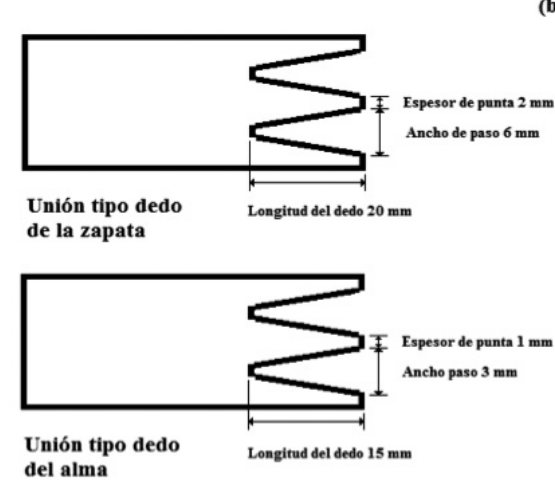

(c)

del alma

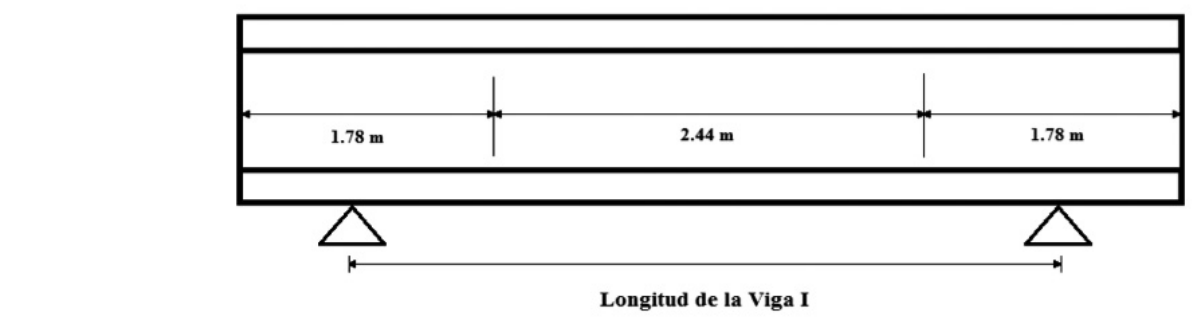

(b)
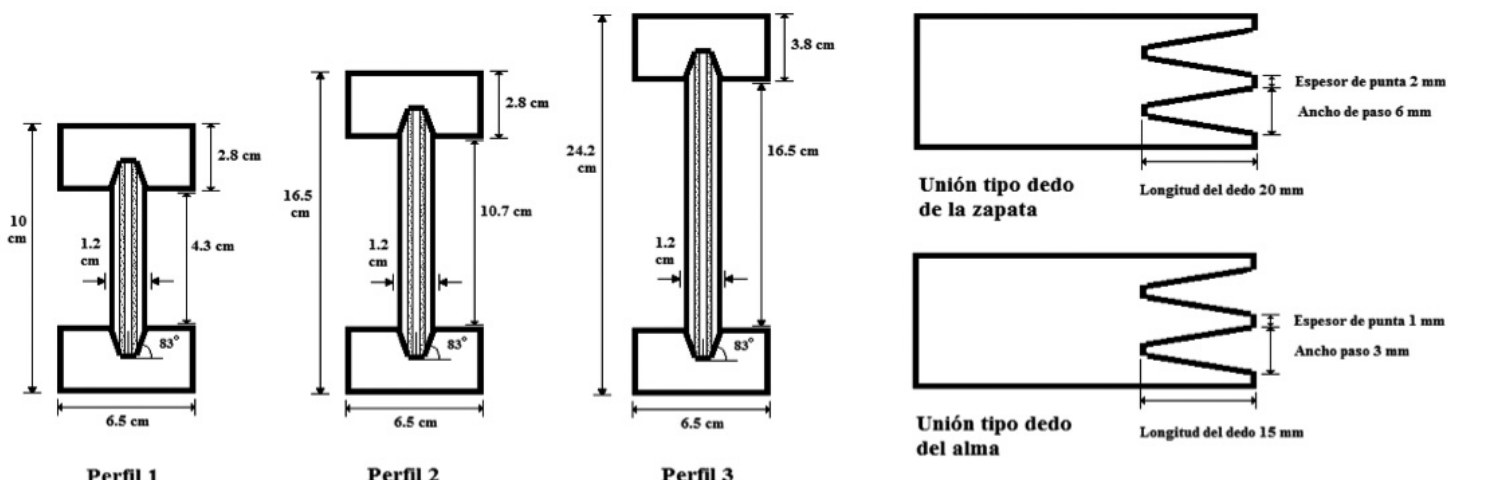

Figura I. Diseño utilizado en las vigas I, (a) perfil I, 2 y 3, (b) unión de dedo utilizada en el alma y la zapata de las vigas, (c) diseño de las vigas I.

muertos, nudos con diámetro mayor a $2,5 \mathrm{~cm}$, rajaduras y madera con ataque de hongos e insectos, hasta dejar longitudes mínimas de $30 \mathrm{~cm}$. Los extremos se unieron utilizando uniones tipo dedo (figura lb) hasta alcanzar una longitud de 6,2 m, usando adhesivo de urea formaldehído. Posteriormente las piezas fueron dimensionadas, cepilladas y molduradas para formar el canal donde se unen con el alma (figura la). Por último, se unieron las zapatas con el alma utilizando urea-formaldehído y una presión de 20 PSI por ocho horas a temperatura ambiente.

\section{Clasificación de vigas}

El proceso de clasificación de la vigas se detalla en Moya et al. (2013). Las vigas se clasificaron visualmente en dos calidades ( $\mathrm{A}$ y B) considerando la cantidad de uniones y su ubicación en las zapatas, la cantidad y el tamaño de los nudos tanto en las zapatas como en el alma, la ubicación de las uniones en el alma, la presencia de grano inclinado en las zapatas y la presencia de pudriciones.
Ensayos mecánicos y determinación de la densidad y el peso de las vigas

Las vigas fueron probadas en flexión estática con la ayuda de una máquina universal de pruebas marca Tinius Olsen con capacidad para 60 toneladas. Para las condiciones de separación entre apoyos, velocidad de carga y determinación de deflexión, se siguieron los procedimientos de la norma europea EN 408 (2003). Mediante estos ensayos se determinó el módulo de elasticidad (MOE) y el módulo de ruptura (MOR). Para mayor detalle consultar Moya et al. (2013). Una vez ensayadas las vigas en flexión, se tomó una muestra de $5 \mathrm{~cm}$ de longitud en la sección transversal, se pesó y se determinó su volumen, para así calcular la densidad de la viga por medio de la relación de la madera entre el volumen. Posteriormente se utilizó este valor para calcular el peso total de la viga, considerando su volumen entre los dos puntos de apoyo. 


\section{Derivación de los esfuerzos de diseño}

se establecieron para cada perfil de viga (I, 2 y 3) y calidad viga ( $A$ y B), para los cuales se muestra su funcionalidad en el uso de entrepisos y techos (figura 2). En la derivación de los esfuerzos de diseño, se analizó la viga estructuralmente en tres tipos de aspectos: (i) capacidad a flexión, (ii) capacidad a cortante y (iii) deflexión en el centro del claro. La capacidad a flexión consiste en la determinación de la máxima carga distribuida que puede soportar una viga de longitud variable y de varios peraltes (perfiles), tomando en cuenta únicamente el esfuerzo de rotura en la fibra extrema. La capacidad a cortante consiste en la determinación de la máxima carga distribuida que puede soportar una viga de longitud variable y de varios peraltes, tomando en cuenta únicamente el esfuerzo de máximo de cortante en los apoyos. Y, finalmente, la deflexión en el centro del claro consiste en la determinación de la máxima carga distribuida que puede soportar una viga de longitud variable y de varios peraltes, tomando en cuenta únicamente la deflexión máxima.

Se siguieron los siguientes pasos: (i) cálculo del momento del peso de la viga (Mpp) (Ecuación I), (ii) con dicho valor se cálculo el momento de ruptura (Mrup) (Ecuación 2), y (iii) se calculó el MORc (Ecuación 3), mediante el Mrup ya que este es el valor señalado como adecuado en el código sísmico de Costa Rica (CFIA 2002).

$$
\begin{aligned}
& \text { Mpp }=\frac{\text { Peso }_{\text {viga }} * L}{8}(\text { Ecuación I) } \\
& \text { Mrup }=\frac{C_{\text {máx }}}{23} * \frac{L}{-}+M_{p p}(\text { Ecuación 2) } \\
& \text { MORc }=\frac{M_{\text {rup }} *(H / 2)}{I}(\text { Ecuación 3) }
\end{aligned}
$$

Mpp: momento del peso de la viga $(\mathrm{kg}-\mathrm{cm})$

Mrup: momento de ruptura ( $\mathrm{kg}-\mathrm{cm})$

MORc: módulo de ruptura $\left(\mathrm{Kg} / \mathrm{cm}^{2}\right)$

$\mathrm{C}_{\text {máx }}$ : carga máxima $(\mathrm{Kg})$

L: largo entre los apoyos (cm)

I: momento de inercia $\left(\mathrm{cm}^{4}\right)$

$\mathrm{H}$ : altura de la viga $(\mathrm{cm})$
Con los valores del MORc se realizó una distribución de frecuencias y se seleccionó el $5^{\text {to }}$ percentil como el valor del esfuerzo de flexión paralelo a la fibra, el cual fue utilizado en capacidad de flexión y capacidad de cortante $\left(\mathrm{fm}_{\mathrm{F}}\right.$ y $\mathrm{fm}_{\mathrm{C}}$ respectivamente). Con el valor promedio del MOE y el fm más un factor de duración de carga temporal $(0,8)$ (Arguelles y Arriaga 2000), se obtuvo un esfuerzo a flexión paralelo a la fibra de diseño (Fd), y con este se calculó el MOR de diseño en flexión. En tanto que en la capacidad de cortante mediante el uso del $\mathrm{fm}_{c}$ y un factor de corrección de 0,5 (Arguelles y Arriaga, 2000), se calculó un esfuerzo a cortante (Fv), y con este valor se obtuvo el valor final de cortante $(\mathrm{Vu})$. Finalmente, para la deflexión en el centro del claro se tomó el MOE promedio de las vigas en cada categoría y calidad y la inercia (American Wood Council 2005) para calcular la rigidez a flexión (E*I). Para este caso, la deformación permitida que se utilizó para entrepisos corresponde a la luz entre apoyos entre 360, para el caso de techos con revestimientos corresponde a la luz entre apoyos entre 240.

\section{Tabla de diseño de entrepisos}

Con el fin de mostrar la utilidad de los valores de diseño, se presentan las figuras para tres tipos de separación entre vigas (S, figura 2), de 40, 60 y 80 cm, comúnmente utilizadas en Costa Rica. En la obtención de estas figuras se determinó la longitud máxima permisible de diseño para cada perfil y calidad. Dicha longitud se calculó para los tres posibles esfuerzos que se presenten en las vigas: flexión, cortante y rigidez de deformación, y se seleccionó el menor de los tres para utilizarlo en las tablas de diseño. A este valor se le aplicaron diferentes usos para cargas temporales unitarias mínimas establecidas en el código sísmico de Costa Rica (CFIA 2002), además de sobrecargas adicionales con rangos de 50 a 150 cada $25 \mathrm{~kg} / \mathrm{m}^{2}$, para posibles usos de vigas en entrepiso (cuadro I).

\section{Tabla de diseño de techos}

Con el fin de mostrar la utilidad de los valores de diseño, se presentan las figuras y su correspondiente tabla para los diferentes tipos de separación entre vigas (S, figura 2), de 40,60 y $80 \mathrm{~cm}$ para los perfiles I, 2 y 3, además de una separación adicional de 100 $\mathrm{cm}$ para el perfil 3. En la obtención de estas figuras se determinó la longitud máxima de diseño para 


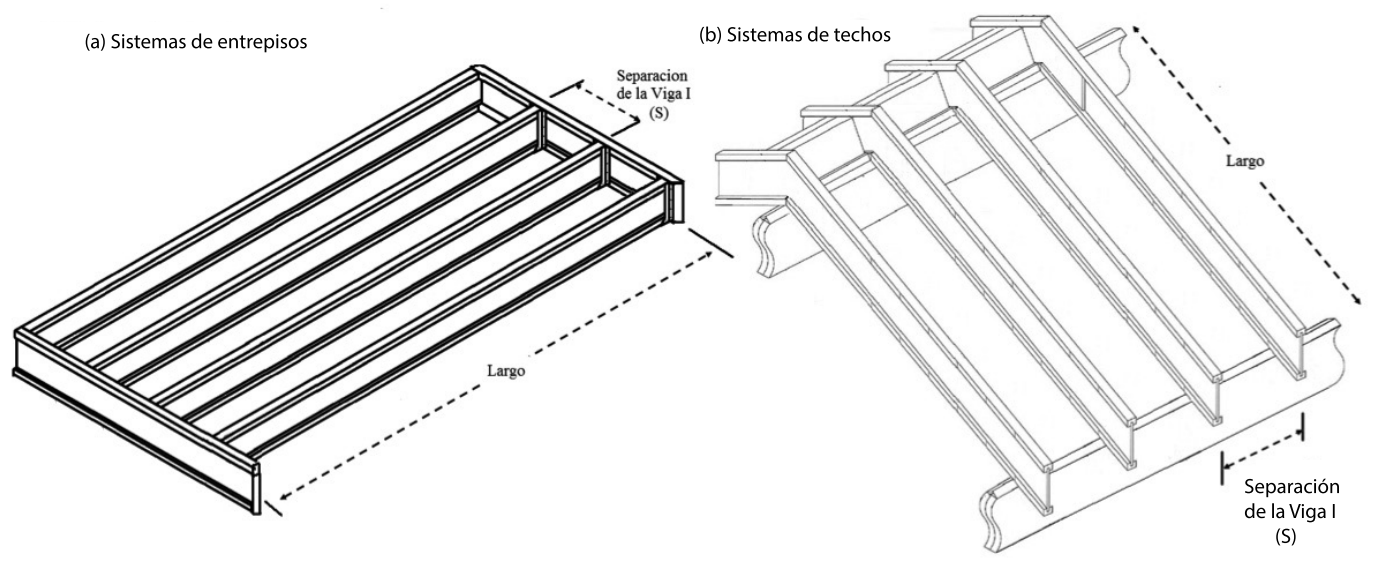

Figura 2. Uso de las vigas en entrepisos y techos.

Cuadro I. Descripción de uso para las cargas temporales unitarias mínimas establecidas en el código sísmico de Costa Rica para uso en entrepisos.

\begin{tabular}{|c|c|}
\hline $\begin{array}{l}\text { Uso para Cargas temporales unitarias mínimas } \\
\text { de acuerdo al CSCR }\end{array}$ & CT Carga Temporal kg/m² \\
\hline $\begin{array}{l}\text { Habitación (casas de habitación, apartamentos, viviendas, dormitorios, cuartos de hotel, edificios } \\
\text { para internados en escuelas, cuarteles, cárceles, correccionales, hospitales y similares). Azoteas } \\
\text { con pendiente inferior a } 5 \text { por ciento. }\end{array}$ & 200 \\
\hline Oficinas, despachos, laboratorios, salones de lectura, aulas, salas de juego y similares. & 250 \\
\hline $\begin{array}{l}\text { Voladizos en vía pública (marquesinas, balcones y similares). Garajes y aparcamientos (para } \\
\text { automóviles exclusivamente). }\end{array}$ & 300 \\
\hline $\begin{array}{l}\text { Escaleras, rampas, vestíbulos, pasajes de libre acceso al público. Lugares de reunión con asientos } \\
\text { fijos, templos, cines, teatros, gimnasios, etc. }\end{array}$ & 350 \\
\hline $\begin{array}{l}\text { Lugares de reunión desprovistos de asientos fijos, estadios, salones de baile, bibliotecas, salones } \\
\text { de archivo. Comercios, bodegas y fábricas de mercancía ligera. }\end{array}$ & 450 \\
\hline Comercios, bodegas y fábricas de mercancías con peso intermedio. & 550 \\
\hline Comercios, bodegas y fábricas de mercancía pesada. & 700 \\
\hline
\end{tabular}

cada perfil y calidad. Dicha longitud se calculó para los tres posibles esfuerzos que se presenten en las vigas: flexión, cortante y rigidez de deformación, y se seleccionó el menor de los tres para utilizarlo en las tablas de diseño. A este valor se le aplicó una carga temporal unitaria mínima de $40 \mathrm{~kg} / \mathrm{m}^{2}$ establecida en el código sísmico de Costa Rica (CFIA 2002), como uso para techos de fibrocemento, lámina de acero galvanizado y otros, además de sobrecargas adicionales con rangos de 20 a 120 cada $20 \mathrm{~kg} / \mathrm{m}^{2}$.

\section{Resultados y análisis}

En el cuadro 2 se observan los esfuerzos de flexión, cortante y la rigidez a flexión de las vigas I derivados de los ensayos. Se utilizaron dichos valores para determinar la longitud máxima permisible para cada viga en flexión, cortante y deformación respectivamente. Los esfuerzos a flexión y cortante se determinan para cada perfil de viga y calidad, mientras que la rigidez a flexión solo para cada perfil. Los resultados obtenidos demuestran que las vigas 
Cuadro 2.Valores de diseño obtenidos para las vigas I de G. arborea.

\begin{tabular}{|c|c|c|c|c|c|c|c|}
\hline Perfil & Calidad & Mpp $(\mathrm{kg}-\mathrm{cm})$ & $\begin{array}{c}\text { Mrup }(\mathrm{kg}- \\
\mathrm{cm})\end{array}$ & $\begin{array}{c}\text { MORc } \\
(\mathrm{MPa})\end{array}$ & $\mathrm{Fd}\left(\mathrm{kg} / \mathrm{cm}^{2}\right)$ & $\mathrm{Fv}\left(\mathrm{kg} / \mathrm{cm}^{2}\right)$ & $\begin{array}{c}\text { E*| } \\
\left(\mathrm{kg}-\mathrm{cm}^{2}\right)\end{array}$ \\
\hline I & A & 126,0 & 20674,9 & 20,6 & 147,5 & 92,2 & 35892503,4 \\
\hline 2 & B & 125,8 & 12480,6 & 12,4 & 67,5 & 42,2 & \\
\hline & A & 411,5 & 39084,5 & 17,2 & 118,6 & 74,1 & 126099372,9 \\
\hline 3 & B & 409,2 & 27322,1 & 12,1 & 60,1 & 37,6 & \\
\hline & B & 1243,9 & 78783,2 & 16,5 & 120,1 & 75,1 & 302787437,1 \\
\hline
\end{tabular}

Nota: Mpp: momento del peso de la viga, Mrup: momento de ruptura, MORc: módulo de ruptura corregido, Fd: esfuerzo a flexión, Fv: esfuerzo a cortante, E**: rigidez a flexión.

perfil I de calidad A poseen los valores de esfuerzo a flexión y cortante más altos ( 147,5 y $\left.92,2 \mathrm{~kg} / \mathrm{cm}^{2}\right)$, seguidos por las vigas perfil 3 y 2 de calidad A. De modo que las vigas de calidad $B$ poseen los valores de esfuerzo más bajos, siendo dentro de este grupo las vigas perfil 3 las que poseen los valores más altos, con $80,8 \mathrm{~kg} / \mathrm{cm}^{2}$ para flexión y $50,5 \mathrm{~kg} /$ $\mathrm{cm}^{2}$ para cortante, seguidos de las vigas perfil I y 2 respectivamente. Con respecto a la rigidez a flexión, las vigas perfil 3 son las que poseen el valor más alto, con 302787437, I kg-cm²; seguidas de los perfiles 2 y I, con 126099372,9 y 35892503,4 kg-cm² respectivamente.

De acuerdo con Keenan et al. (1987) y su clasificación propuesta para el Grupo Andino para las maderas de los países de América del Sur, podemos clasificar a $G$. arborea $(0,45)$ en el grupo $C$, de las especies con densidad básica de 0,40 a 0,55, siendo las especies de menor resistencia y rigidez. Para las especies ubicadas en este grupo se presentan valores de esfuerzos admisibles en flexión de $100 \mathrm{~kg} /$ $\mathrm{cm}^{2}$, el cual es menor que el obtenido para las vigas de calidad A de este estudio, sin embargo, es mayor que el obtenido en las vigas de calidad B. Además se presenta un valor de esfuerzo de cortante de 8 $\mathrm{kg} / \mathrm{m}^{2}$ para las especies del grupo C, valor mucho menor que el reportado para $G$. arborea en este estudio, de 92 a $74 \mathrm{~kg} / \mathrm{m}^{2}$ para vigas de calidad A y de 37 a $50 \mathrm{~kg} / \mathrm{m}^{2}$ para vigas de calidad B.

La figura 3 representa gráficamente las longitudes máximas permisibles para las vigas I de los tres perfiles y calidades de G. arborea para utilizar en entrepisos con separaciones de 40, 60 y 80 cm, comúnmente utilizados en Costa Rica. Por ejemplo, en vigas de perfil 3 y de calidad A con una carga de $300 \mathrm{~kg} / \mathrm{m}^{2}$ y separación de $40 \mathrm{~cm}$ entre vigas, es necesario utilizar longitudes máximas de aproximadamente 4,0 metros. En tanto que al usar una separación de $60 \mathrm{~cm}$, la longitud permisible de separación disminuye a 3,6 metros. Pero si la separación se aumenta a $80 \mathrm{~cm}$ entre vigas, la longitud máxima disminuye a aproximadamente 3,2 metros.

Cuando se utiliza el perfil 2 (altura de $16,5 \mathrm{~cm}$ ), en las mismas distancias de separación de vigas (40, 60 y $80 \mathrm{~cm}$ ), las longitudes máximas permisibles serían de aproximadamente 3,9 m, 2,6 m y 2,4 m respectivamente. En las vigas de perfil I ( $10 \mathrm{~cm}$ de altura), las longitudes permisibles serían las más bajas, de 1,9 m para la separación de $40 \mathrm{~cm}$, de 1,70 m en la separación de $60 \mathrm{~cm}$ y de 1,55 m en la separación de $80 \mathrm{~cm}$ (Figura 3a). De modo que las vigas perfil 3 de las tres separaciones y las vigas perfil 2 de $40 \mathrm{~cm}$ son las que se pueden ubicar a mayores longitudes, con cargas que van de los 200 a los $900 \mathrm{~kg} / \mathrm{m}^{2}$.

En tanto que cuando se utilizan vigas de calidad B usando este mismo tipo de carga $\left(300 \mathrm{~kg} / \mathrm{m}^{2}\right)$, las longitudes permisibles son menores a las presentadas por las vigas de calidad A (figura 4b). Para las vigas de perfil 3, en las separaciones de 40,60 y 80 $\mathrm{cm}$, las longitudes permisibles son de aproximadamente 3,7 m, 3,0 m y 2,6 m respectivamente. Dichas distancias son menores en comparación con las obtenidas para las vigas de calidad A para las mismas distancias de separación (figura 3b). 

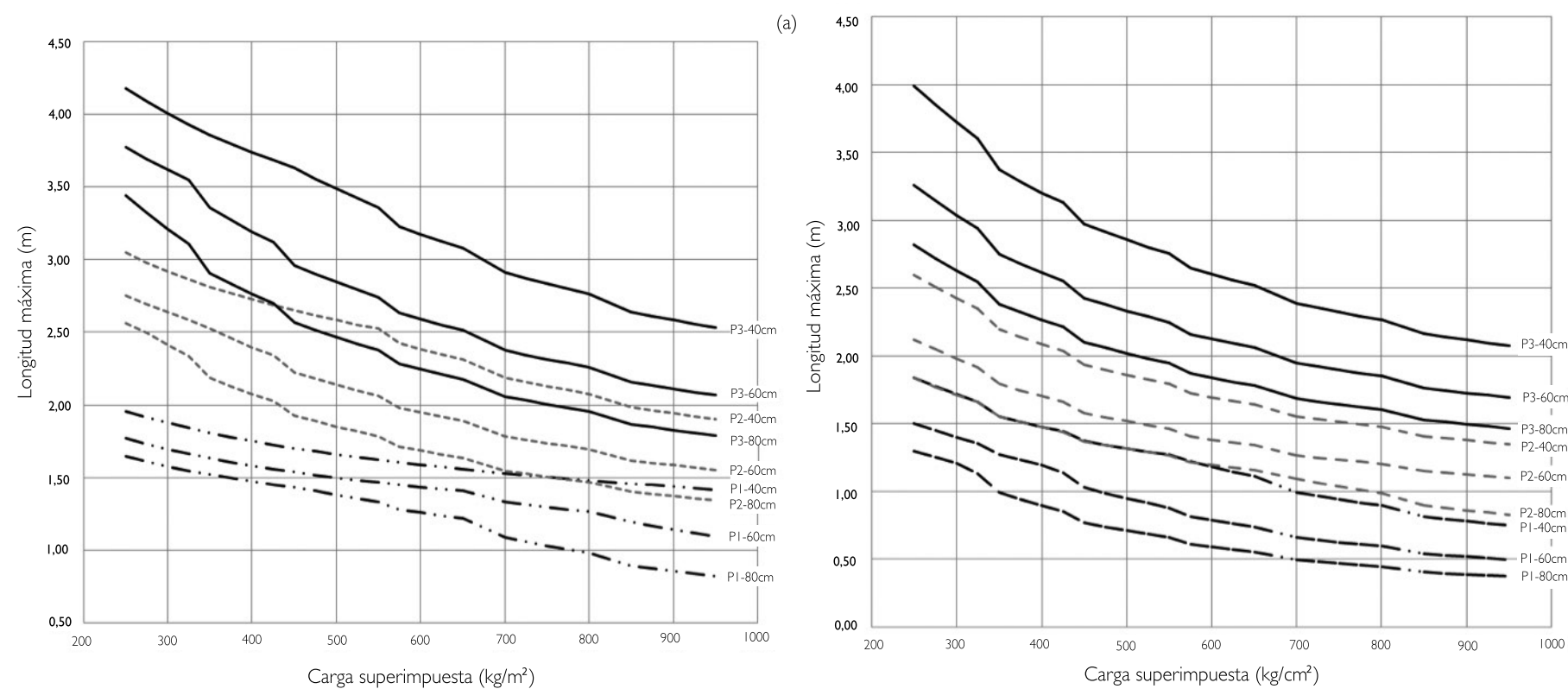

Figura 3. Longitudes máximas permisibles según carga superimpuesta para el uso de vigas I de calidad A (a) y calidad B (b) en entrepisos.
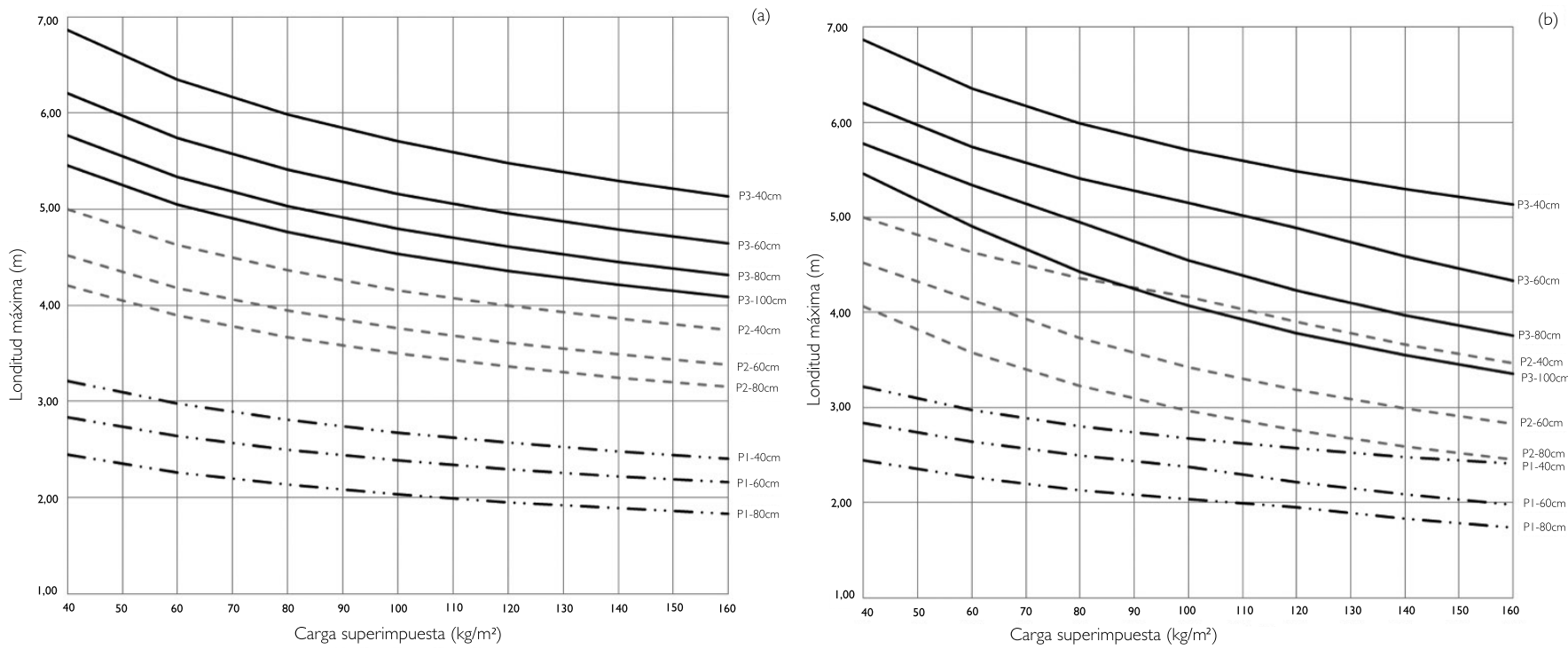

Figura 4. Longitudes máximas permisibles según carga superimpuesta para el uso de vigas I de calidad A (a) y calidad (b) B en techos.

La determinación gráfica de la longitud permisible para utilizar las vigas I en techos se muestra en la figura 4. En este caso, al igual que el uso en entrepisos, las vigas de perfil 3 son las que poseen las mayores longitudes permisibles, pero a diferencia de ese uso, la calidad de las vigas tiene poco efecto en las longitudes permisibles (figura $4 a$ y $4 b$ ). Por ejemplo, para vigas perfil 3, si la carga en el techo es de 60 $\mathrm{kg} / \mathrm{m}^{2}$ utilizando vigas de calidad A y separaciones de
40, la longitud permisible es aproximadamente de 6,4 m, y utilizando el mismo perfil de viga, la misma separación de viga y la misma carga, para vigas de calidad B la longitud permisible es nuevamente 6,4 $m$. Dicho resultado indica que una separación de las vigas por calidad en este tipo de uso no es conveniente para esta especie y para las condiciones que se están utilizando para fabricar las vigas. 


\section{Conclusiones}

Los resultados obtenidos muestran que es posible construir vigas I con madera proveniente de plantaciones de rápido crecimiento de $G$. arborea en tres posibles alturas, de $10 \mathrm{~cm}, 16,5 \mathrm{~cm}$ y 24,2 $\mathrm{cm}$. Dichos perfiles permiten obtener valores de resistencia similares a los de otras especies que se utilizan en la elaboración de vigas I.

Los valores de los esfuerzos en flexión y cortante obtenidos demuestran la necesidad de clasificar las vigas I por calidades. Las vigas de calidad A presentan esfuerzos en flexión mayores a los reportados para las especies del grupo $C$ (especies con densidades básicas entre 0,40 y 0,55), de la clasificación del Grupo Andino, la cual señala esfuerzos en flexión admisibles de $100 \mathrm{~kg} / \mathrm{cm}^{2}$; caso contrario al de las vigas de calidad $\mathrm{B}$, cuyos resultados presentan esfuerzos en flexión menores a los $100 \mathrm{~kg} / \mathrm{cm}^{2}$.

La categorización de las vigas I por perfiles y calidades permite obtener un mejor desempeño cuando estas se utilizan en entrepisos y techos. Las vigas de calidad A presentan mejor resistencia en deflexión, valores de diseño y soporte de cargas. Además, en el uso de las vigas en entrepisos, estas presentaron mayores longitudes permisibles para una misma carga y separación en comparación con las vigas de calidad B. Pero la clasificación por calidades cuando las vigas se usan en techos no presentó diferencias en cuanto a longitudes permisibles, para una misma carga y separación, de forma tal que para el uso en techos y para las condiciones de fabricación utilizadas en este estudio no es necesario realizar una clasificación de calidad para las vigas I de G. arborea.

\section{Agradecimientos}

Los autores agradecen a la Vicerrectoría de Investigación y Extensión del Instituto Tecnológico de Costa Rica (ITCR), así como a Maderas Cultivadas de Costa Rica por suplir y fabricar las vigas I utilizadas en el estudio.

\section{Bibliografía}

American Wood Council. (2005). National Design Specification for Wood Construction. Leesburg, VA. USA.

Arguelles, R., Arriaga, F. (2000). Estructuras de madera, diseño y cálculo. Asociación de Investigación Técnica de la Industria de la Madera y Corcho (AITIM), $2^{\text {da }}$ edición, Madrid. España.

Aydin, I., Colak, S., Colakoglu, G. \& Salih, E. (2004). A comparative study on some physical and mechanical properties of laminated veneer lumber ( $\mathrm{LVL}$ ) produced from beech (Fagus orientalis Lipsky) and eucalyptus (Eucalyptus camaldulensis Dehn) veneers. Holz Roh Werkst 62: 21 8-220.

Colegio Federado de Ingenieros y Arquitectos de Costa Rica (CFIA). (2002). Código sísmico de Costa Rica. Cartago. C.R.: Editorial Tecnológica.

Erskinea, P.D., David Lamba, D., Bristow, M. (2006). Tree species diversity and ecosystem function: Can tropical multi-species plantations generate greater productivity? Forest Ecol Manag 233: 205-210.

European Standard (2003). Timber structures. Structural timber and glued laminated timber. Determination of some physical and mechanical properties (EN 408). Madrid.

González, G., Moya, R., Monge, F., Córdoba, R. \& Coto, J. (2004). Evaluating the strength of finger-joined lumber of Gmelina arborea in Costa Rica. New Forests 28: 319-323.

Keenan, F.J. \& Tejada, M. ( 1987). Maderas tropicales como material de construcción en los países del grupo andino de América del Sur. Ottawa, Ont:: CIID.

Lam, F. (200 I). Modern structural wood products. Prog Struc Eng Mater 3: 238-245.

Leichti, R.J., Falk, R.H. \& Laufenberg, T.L. (1990). Prefabricated wood I-joist: an industry overview. Forest Prod J. 40(3): I 5-20.

Maloney, T.M. (1996). The family of wood composite materials. Forest Prod J 46(2): 19-26.

Moya, R. (2004). Wood of Gmelina arborea in Costa Rica. New Forest 28: 299-307.

Moya, R., Tenorio, C., Carranza, M., Camacho, D. \& Quesada, H. (20।3). Structural performance of I-beam fabricated from a fast-growing tree, Gmelina arborea. Journal of Tropical Forest Science 25(2): | $51-156$.

Sasaki, H., Wan, Q. \& Kawai, S. (1993). Laminated Veneer Lumber and Composite Beams produced from tropical hardwood thinning. Current Jap Mat Res I 1: 55-66.

Tenorio, C. \& Moya, R. (20I I). A comparative study on physical and mechanical properties of laminated veneers lumber and plywood panels made of wood from Gmelina arborea trees growing in fast condition. J Wood Sci 57(2).

Tenorio, C., Moya, R. \& Camacho, D. (2012). Propiedades físicomecánicas de tableros terciados construidos con especies tropicales de plantaciones para uso estructural. Cerne I8(2): 317-325. 